\title{
The Effect of Silk Membrane Plus 3\% 4-hexylresorcinol on Guided Bone Regeneration in a Rabbit Calvarial Defect Model
}

\author{
Hyun Seok ${ }^{1 *}$, Sang-Woon Lee ${ }^{1}$, Seong-Gon $\mathrm{Kim}^{1}$, Dong-hyun $\mathrm{Seo}^{2}$, Han Sung Kim² ${ }^{2}$ Hae Yong Kweon ${ }^{3}$, \\ You-Young Jo ${ }^{3}$, Tae Yeon Kang ${ }^{4}$, Myung-Jin Lee ${ }^{4}$, and Weon-Sik Chae ${ }^{4}$ \\ ${ }^{I}$ Department of Oral and Maxillofacial surgery, College of Dentistry, Gangneung-Wonju National University, Gang neung, Korea \\ ${ }^{2}$ Department of Biomedical Engineering, Institute of Medical Engineering and Yonsei-Fraunhofer Medical, Device Laboratory, Yonsei \\ University, Wonju, Gangwon 220-710, Republic of Korea \\ ${ }^{3}$ Sericultural \& Apicultural Materials Division, National Academy of Agricultural Science, Suwon, 441-707, Korea \\ ${ }^{4}$ Gangneung Center, Korea Basic Science Institute, Gangneung 210-702, Korea
}

\section{Abstract}

The objective of this study was to evaluate the bone regeneration capability of silk membrane plus 3\% 4-hexylresorcinol (3\% 4-HR plus SM) in a rabbit calvarial defect model. Twenty New Zealand white rabbits were used in this study. Bilateral round shaped defects were created in the parietal bone (diameter: $8.0 \mathrm{~mm}$ ). And the defects were covered with (1) $3 \% 4-\mathrm{HR}$ plus SM, (2) collagen membrane (CM), (3) no graft material. After surgery, the animals were sacrificed at 4 weeks and 8 weeks. Bone regeneration was analyzed in each section by micro-computerized tomography $(\mu-\mathrm{CT})$. And Hematoxylin and eosin stains were used for histological analysis. As measured by $\mu$-CT analysis 4 weeks after surgery, the average of new bone formation in animals treated with $3 \% 4$-HR plus SM was greater than that of animals treated with CM. and the difference was statistically significant. And well organized lamella bones were observed in the histological view of the 3\% 4-HR plus SM group. Therefore, more bone regeneration was seen in animals treated with 3\% 4-HR plus SM than in those treated with $\mathrm{CM}$ or uncovered control.

(c) 2013 The Korean Society of Sericultural Sciences Int. J. Indust. Entomol. 27(1), 209-217 (2013)

Received : 9 Sep 2013

Accepted : 14 Sep 2013

Keywords:

4-Hexylresorcinol, Guided bone regeneration, Silk membrane

\section{Introduction}

The concept of guided bone regeneration (GBR) employs a barrier membrane to prevent ingrowth of soft tissue cells but allow migration of angiogenic and osteogenic cells originating from bone marrow into bone defects (Schenk et al. 1994). Barrier membranes provide a physical barrier, a substrate to stabilize bone graft materials and blood clots and a space for osteoblasts to regenerate bone without interference from soft tissue cells (Buser et al. 2007). Characteristics of a GBR membrane include biocompatibility, stability sufficient to maintain a space for ingrowth, occlusivity to prevent fibroblast and bacteria infiltration, tissue integrability, and clinical manageability that is practical to applicate bone defect during surgery (Rakhmatia et

\section{*Corresponding author.}

Hyun Seok

Department of Oral and Maxillofacial Surgery, College of Dentistry, Gangneung-Wonju National University, 7 Jukheon-gil, Gangneung, Gangwon-Do, 210-702, Korea

Tel: +82-33-640-3139 / FAX: +82-33-642-3113

E-mail: sok8585@hanmail.net 
al. 2013).

GBR membranes can be divided into nonresorbable and bioresorbable categories. Nonresorbable membrans are composed of expanded polytetrafluoroethylene (e-PTFE) or titanium mesh. These membranes provide good biocompatibility, space maintenance for bone growth, and clinical manageability. However it is not bioresorbable, so surgical intervention for removal of membrane is required after bone regeneration. Also it can happen membrane exposure after GBR and it can often lead to local infection and GBR failure (Gotfredsen et al. 1993). Contrast, bioresorbable membranes do not require surgical removal (Gottlow 1993). It has been shown that bioresorbable membranes and nonresorbable barriers promote similar levels of bone formation (Imbronito et al. 2002). However, the high cost of bioresorbable and nonresorbable membrane constrains their routine use. Both clinicians and patients would benefit from a new membrane that is composed of the relatively inexpensive materials, and that performs similar to or better than existing products.

Silk is a fibrous protein composed of sericin and fibroin that is produced by Bombyx mori silkworm (Cao et al. 2009). For human applications, silk protein is usually degummed to remove sericin because it may trigger an inflammatory reaction (Altman et al. 2003). Silk fibroin causes only minimal inflammatory reactions and it can be used safely without side effects (Aramwit et al. 2009). However, silk fibroin membrane (SFM) is difficult to apply clinically because it is less flexible than silk and easily breaks away (Lee et al. 2012). In this study, we applied silk membrane (SM) that had not been degummed to remove sericin. Silk is extremely biocompatible, highly permeable to oxygen and water vapor, and biodegraded without toxicity (Minoura et al. 1990; Um et al. 2001; Dal Pra et al. 2005). Because of its superior mechanical properties (Gosline et al. 1986), it is used in biomedical application such as drug-delivery devices, tissue engineering scaffolds, vessels, and artificial tympanic membranes (Sofia et al. 2000; Arai et al. 2003, Kim et al. 2004, Kim et al. 2010). Previously study have shown that SM facilitates new bone formation in artificial bone defects (Song et al. 2011; Lee et al. 2012).

Alkylresorcinols are natural non-isoprenoid lipids with antioxidant and antimutagenic properties that are found in various plants and microorganisms (Kozubek et al. 1999). The alkylresorcinol 4-hexylresorcinol (4-HR) is an antiparasitic and antiseptic agent that has been used to inhibit enzymatic browning in apples and lobsters. It does not produce toxic effects and can be safely used as a food additive (Iyidogan et al. 2004; LópezCaballero et al. 2007). Historically, 4-HR has been used as an antiparasitic agent in children (Rabbani et al. 1985), and as an antibiotic to treat typhoid carriers. It is currently used as an ingredient in oral antiseptic solutions (Young et al. 1927).

We hypothesized that combining 4-HR's antiseptic activity with silk membrane would compare favorably to collagen membrane (CM) in facilitating new bone formation in bone defects. In this study we evaluated the bone regeneration capability of silk membrane plus 3\% 4-hexylresorcinol (3\% 4-HR plus SM) in a rabbit calvarial defect model. New bone formation was assessed with microscopic computerized tomography $(\mu-\mathrm{CT})$ and histomorphometry.

\section{Material and methods}

\section{Scanning electron microscope imaging, X-ray diffraction, and Attenuated total reflectance Fourier-transform infrared absorbance spectra}

Scanning electron microscope (SEM) imaging was performed with an electron microscope (Hitachi, SU-70). Attenuated total reflectance Fourier transform infrared spectroscopy (ATR-FTIR) measurements were made with a Vertex 80 spectrometer (Bruker Optics, Germany) coupled to a microscope (Hyperion 3000 , Bruker Optics, Germany) equipped with a germanium (Ge) attenuated total reflectance objective lens (ATR 20×) and a liquid nitrogen cooled mercury cadmium telluride (MCT) detector. The spectra were obtained with 256 repeated scans from 600 to 4,000 $\mathrm{cm}^{-1}$ at a resolution of $4 \mathrm{~cm}^{-1}$. X-ray diffraction (XRD) patterns of the samples were recorded with an X-ray diffractometer (PANalytical, X'Pert Pro MPD) using a Cu-K $\alpha(\lambda=1.5418 \AA)$ radiation source and collected in the range of $0-30^{\circ}(2 \theta)$

\section{Animals and surgical procedures}

Twenty 10 week-old New Zealand white rabbits with an average weight of $2.3 \mathrm{~kg}$ (range $2.0-2.5 \mathrm{~kg}$ ) were used in this study. The study was approved by the Institutional Animal Care and Use Committee of the Gangneung-Wonju National 


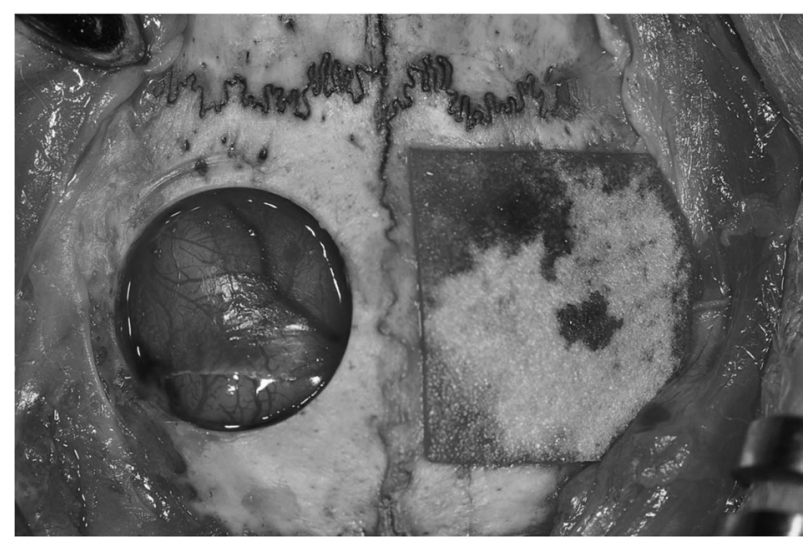

Fig. 1. 3\% 4-HR plus Silk membrane or collagen membrane was grafted into bone defects of the parietal bone. Some defects were left empty. 4-HR; 4-hexylresorcinol.

University, Gangneung, Korea (IACUC GWNU-2012-21).

General anesthesia was administered by intramuscular injection of a combination of $0.5 \mathrm{~mL}$ Tiletamine and Zolazepam (125 mg/ $\mathrm{mL}$; Zoletil; Bayer Korea, Seoul, Korea) and $0.5 \mathrm{~mL}$ Xylazine hydrochloride (10 mg/kg body weight; Rompun; Bayer Korea). The cranium area was shaved and disinfected with povidoneiodine and $2 \%$ lidocaine with epinephrine $(1: 100,000)$ was applied to the cranium area. A longitudinal incision was made from the nasal bone to the occipital protuberance of the skull. Then a midline incision was made in the periosteum. Sharp subperiosteal dissection reflected the pericranium from the outer table of the cranial vault, exposing the parietal bones. A trephine bur was used under saline irrigation to create bilateral calvarial defects in the parietal bones. Two $8 \mathrm{~mm}$ diameter defects were created, one on each side of the midline. The calvarial defects were covered with 3\% 4-HR plus SM or CM (Rapi-GuideTM, Dalim tissen, Seoul; Fig. 1). Some defects were left empty to serve as controls. Defects were randomly assigned to each group. None of the animals received the same grafts in both calvarial defects.

Following the treatment, the pericranium and skin were closed in layers with 3-0 black silk. After surgery, the rabbits received gentamycin at $1 \mathrm{mg} / \mathrm{kg}$ (Kookje, Seoul, Korea) and Pyrin at $0.5 \mathrm{~mL} / \mathrm{kg}$ (Green Cross Veterinary Products, Seoul, Korea) intramuscularly 3 times daily for 3 days.

Each rabbit was individually caged and received food and water. Ten animals were killed at 4 weeks and the other ten at 8 weeks. Specimens were separated and fixed in 10\% formalin. Histologic analysis was done after $\mu$-CT analysis.

\section{$\mu-C T$ analysis}

The Skyscan 1076 (SkyScan 1076; Bruker, Aartselar, Belgium) was used specimens with $\mu$-CT. After calibration, the calvarial specimens were scanned in $0.035-\mathrm{mm}$ thick sections. The scanned images were reconstructed with CT-AN 1.10 software (Bruker, Aartselaar, Belgium ). The calibrated 3-dimensional images were shown in the gross profiles of the specimens. The region of interest (ROI) was set as the initial 8.0 $\mathrm{mm}$ diameter round defect. A threshold level of $25 \%$ of the bone standard was set as recommended by the manufacturer. The ROI of each specimen was analyzed for bone volume (BV).

\section{Histomorphometric evaluation}

At 4 and 8 weeks after surgery, calvarial samples were subjected to dehydration and embedding. The specimens were decalcified with the $5 \%$ nitric acid for 2 weeks and dehydrated in ethyl alcohol and xylene. After the parietal bones were separated through the sagittal suture, they were embedded in paraffin blocks. The paraffin blocks were sliced and stained with Hematoxylin and Eosin. The section showing the widest defect area was selected, along with the cuts from $50 \mu \mathrm{m}$ before and after. Digital images of the selected sections were made with a digital camera (DP-73; Olympus, Tokyo, Japan). The images were analyzed with a Sigma Scan Pro (SPSS, Chicago, IL). Total new bone was calculated as a percentage of the total region of the defect.

\section{Statistical analysis}

SPSS for Windows, ver. 19 (IBM Co., Armonk, NY, USA) was used for statistical analysis, and analysis of varance (ANOVA) and lease significant difference (LSD) were used as post hoc tests for comparison of the samples. The level for statistical significance was set at $p<0.05$.

\section{Results}

\section{SEM, XRD, ATR-FT-IR results}

Fig. 2 shows the ATR-FT-IR spectra of SM, 3\% 4-HR plus $\mathrm{SM}$, and type 1 collagen. The observed spectrum of SM shows 


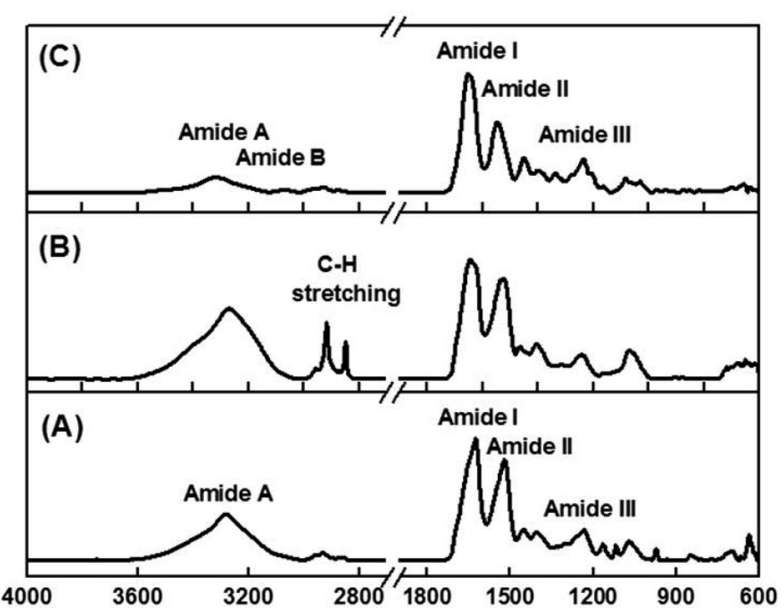

Fig. 2. ATR-FT-IR spectra. (A) Silk membrane; (B) 3\% 4-HR plus silk membrane; and (C) collagen membrane.

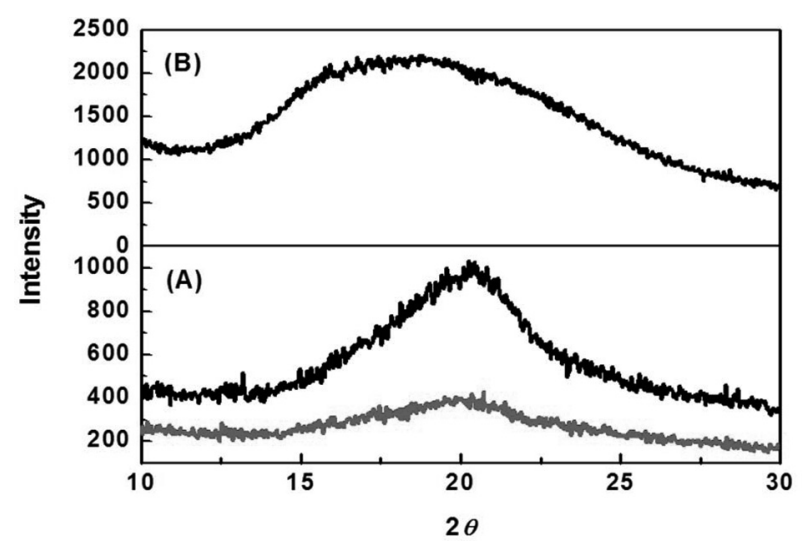

Fig. 3. XRD patterns. (A) Silk membrane (black line) and 3\% 4-HR plus silk membrane (red line). (B) Collagen membrane.

the typical amide absorption bands in all spectral regions (Fig. 2A). The absorption bands at 1625 (amide I) and $1520 \mathrm{~cm}^{-1}$ (amide II) were attributed to a $\beta$-sheet conformation. and the absorption band at $1235 \mathrm{~cm}^{-1}$ (amide III) was attributed to a random coil structure. In the case of SM blended with the 4-HR, vibrational absorptions corresponding to pure 4-HR are seen at 2960,2920, $2860 \mathrm{~cm}^{-1}$, which were attributed to C-H stretching vibration. Fig. 2B shows that the amide band positions and intensities were unchanged by blending with $\mathrm{SM}$ with 4-HR, indicating that 4-HR is well adsorbed into SM. Fig. 2C shows the absorption spectrum of type I collagen. Characteristic absorption bands appeared at 1652 (amide I), 1546 (amide II), and $1236 \mathrm{~cm}^{-1}$ (amide III). Amide A and amide $\mathrm{B}$ bands were found at 3320 and $3080 \mathrm{~cm}^{-1}$, respectively. The amide $\mathrm{B}$ band was only observed in collagen samples.

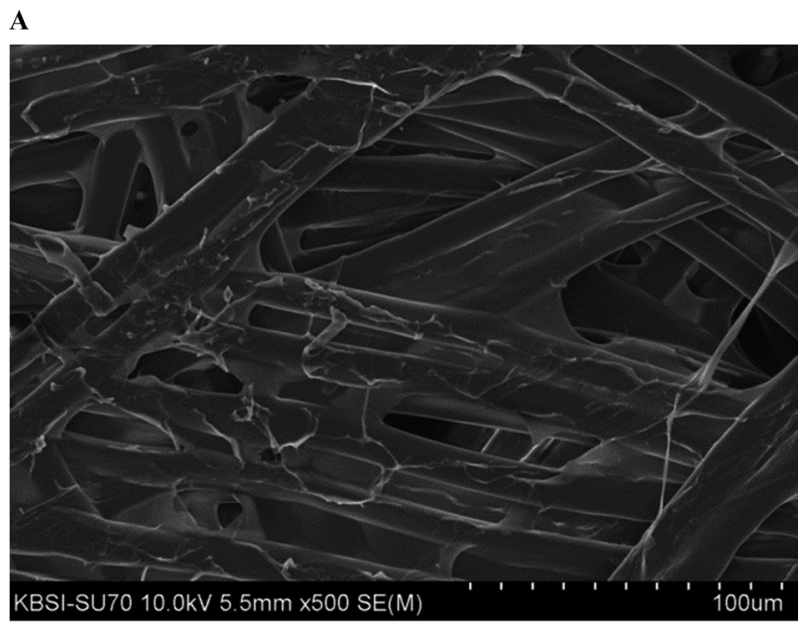

B

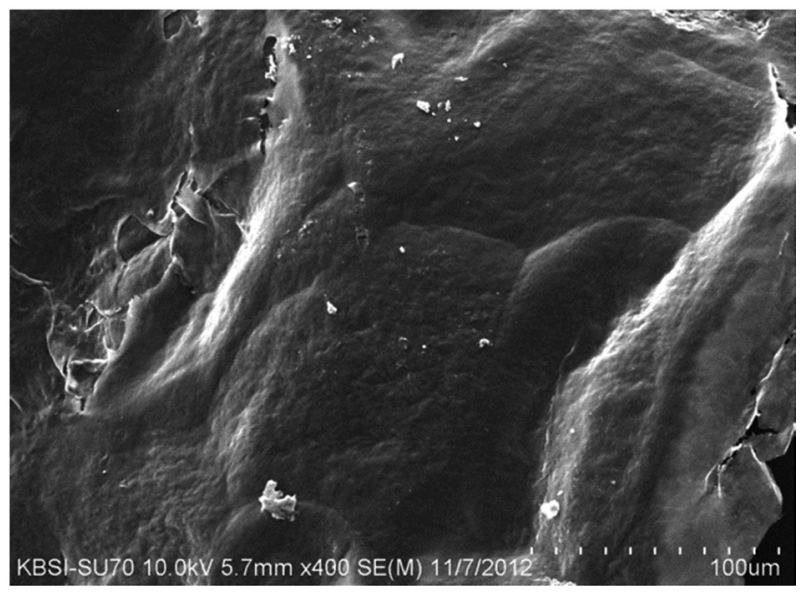

Fig. 4. Scanning microscopic images. (A) 3\% 4-HR plus silk membrane. (B) collagen membrane.

XRD patterns of SM, 3\% 4-HR plus SM, and type I collagen are shown in Fig. 3. SM was characterized by the presence of the broad diffraction peak at $20^{\circ}(2 \theta)$, which was attributed to the crystalline regions of $\beta$-sheet structure (Fig. 3A). Diffraction from 3\% 4-HR plus SM (red line) shows reduced intensity compared to that of pure SM. Indicating that the crystallinity of SM was not changed by 4-HR blending. Fig. 3B shows the XRD patterns of CM. An extensively broadened peak was observed in the range of $10-30^{\circ}$. The broadened diffraction peak can be attributed to the amorphous characteristic of CM.

The SEM image of 3\% 4-HR plus SM demonstrates the cross-linked structure of silk fiber with some glue like-material attached among the fibers (Fig. 4A). The diameter of silk fiber is approximately $100 \mu \mathrm{m}$. CM has a smooth surface but some 
Table 1. Microscopic computerized tomographic analysis

\begin{tabular}{|ccccccc|}
\hline & \multicolumn{3}{c}{ 4 weeks } & \multicolumn{3}{c|}{8 weeks } \\
\hline & Unfilled & CM & $3 \% 4-H R+S M$ & Unfilled & CM & $3 \% 4-H R+$ SM \\
\hline Bone volume $\left(\mathrm{mm}^{3}\right)$ & $5.68 \pm 3.27$ & $9.71 \pm 6.29$ & $12.53 \pm 3.45^{*}$ & $18.85 \pm 8.71$ & $16.53 \pm 13.44$ & $17.31 \pm 6.65$ \\
\hline
\end{tabular}

Silk membrane plus 3\% 4-hexylresorcinol ( $3 \%$ 4-HR + SM), collagen membrane (CM).

${ }^{*} p<0.05$; compared with unfilled control.

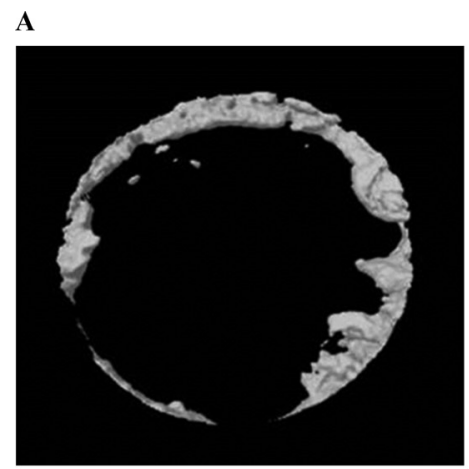

D

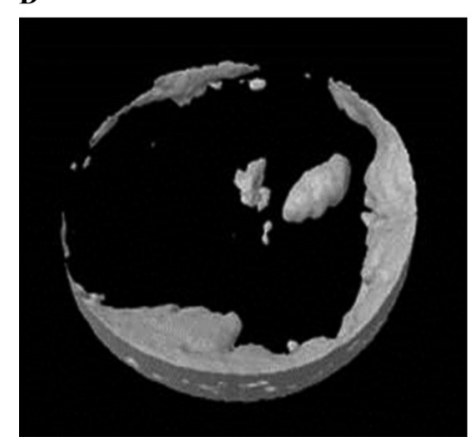

B

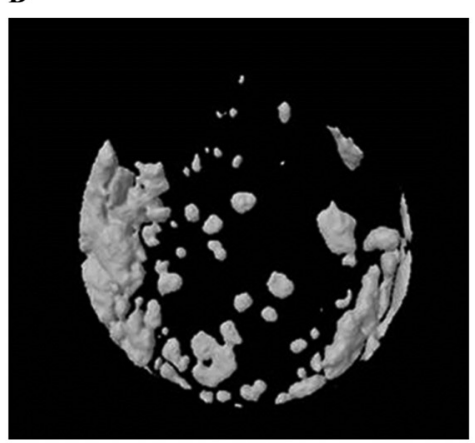

E

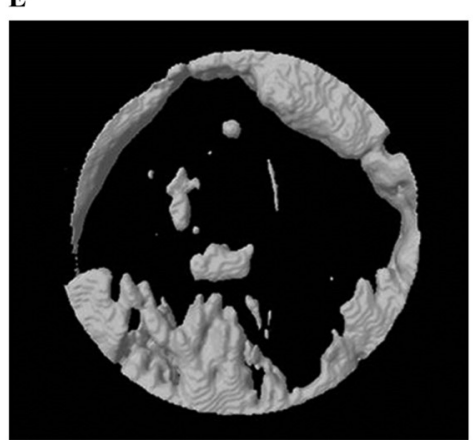

C

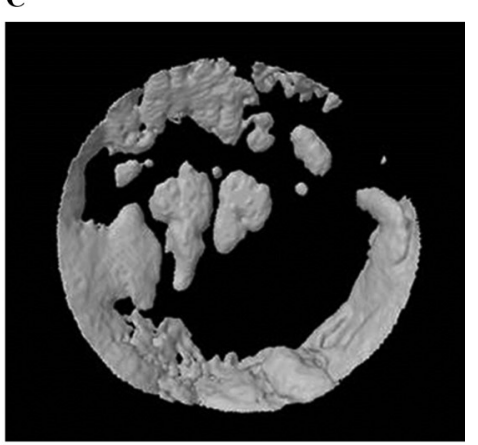

F

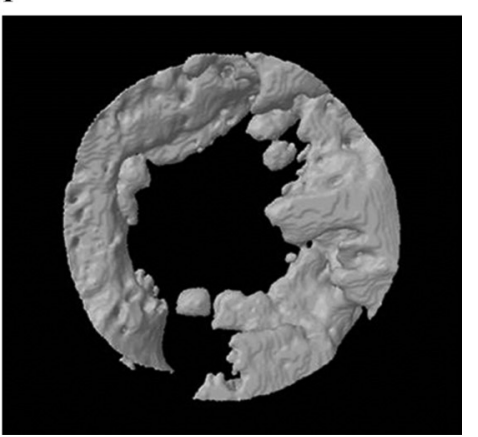

Fig. 5. Micro-computerized tomography. (A) Unfilled control 4 weeks after surgery. (B) Collagen membrane at 4 weeks after surgery. (C) 3\% 4-HR plus silk membrane at 4 weeks after surgery. (D) Unfilled control 8 weeks after surgery. (E) Collagen membrane at 8 weeks after surgery. (F) 3\% 4-HR plus silk membrane 8 weeks after surgery. 4-HR; 4-hexylresorcinol.

regions appear to be torn (Fig. 4B).

\section{$\mu-C T$ analysis}

Results of the $\mu-\mathrm{CT}$ analysis are presented in Table 1. Four weeks after surgery the volume was $12.53 \pm 3.45 \mathrm{~mm}^{3}$ in the $3 \% 4$-HR plus SM group, $9.71 \pm 6.29 \mathrm{~mm}^{3}$ in the CM group, and $5.68 \pm 3.27 \mathrm{~mm}^{3}$ in the unfilled group (Fig. 5). A post hoc analysis showed that the differences between the CM group and any other group were not statistically significant. However, there was a significant difference between the 3\% 4-HR plus SM and unfilled groups $(\mathrm{P}=0.031)$.

The mean bone volume in the 3\% 4-HR plus SM group was higher than the CM group. The bone volume in the 3\% 4-HR plus SM group 8 weeks after surgery was $17.13 \pm 6.65 \mathrm{~mm}^{3}$. That in the CM group was $16.53 \pm 13.44 \mathrm{~mm}^{3}$ and the unfilled group was $18.85 \pm 8.71 \mathrm{~mm}^{3}$ (Fig. 5). There was no statistically significant difference $(\mathrm{P}>0.05)$.

\section{Histomorphometric analysis}

The histomorphometry results are presented in Table 2. Four weeks after surgery total new bone was $8.73 \pm 3.08 \%$ in the unfilled group, $5.29 \pm 0.72 \%$ in the CM group, and $9.39 \pm 1.86 \%$ in the 3\% 4-HR plus SM group (Fig. 6). The differences were not statistically significant $(\mathrm{P}>0.05)$. Eight weeks after surgery 
Table 2. Histomorphometric analysis

\begin{tabular}{|ccccccc|}
\hline & \multicolumn{4}{c}{4 weeks } & \multicolumn{3}{c|}{8 weeks } \\
\hline & Unfilled & CM & $3 \% 4-H R+$ SM & Unfilled & CM & $3 \% 4-H R+$ SM \\
\hline Total new bone $(\%)$ & $8.73 \pm 3.08$ & $5.29 \pm 0.72$ & $9.39 \pm 1.86$ & $31.1 \pm 7.39$ & $36.31 \pm 5.34$ & $38.82 \pm 4.12$ \\
\hline
\end{tabular}

Silk membrane plus 3\% 4-hexylresorcinol (3\% 4-HR + SM), collagen membrane (CM).

A

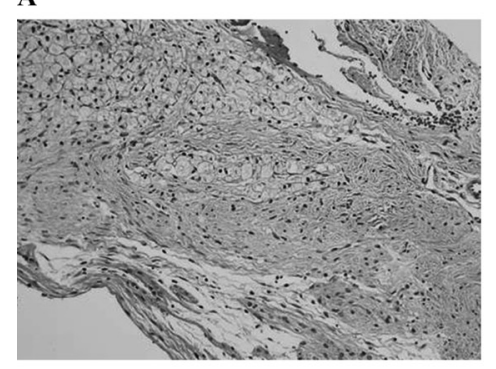

D

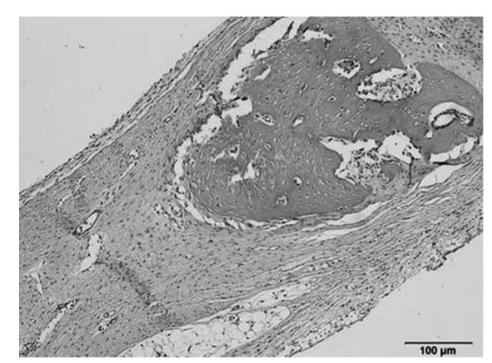

B

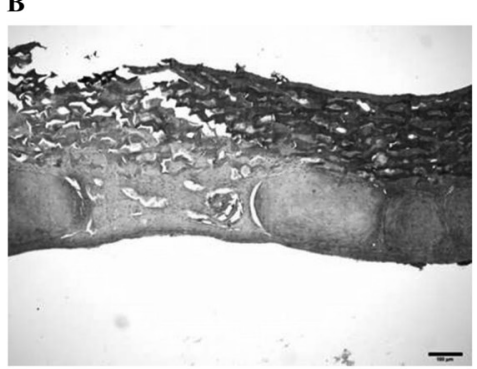

$\mathbf{E}$

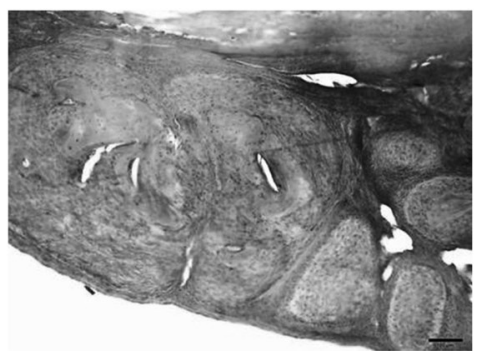

C

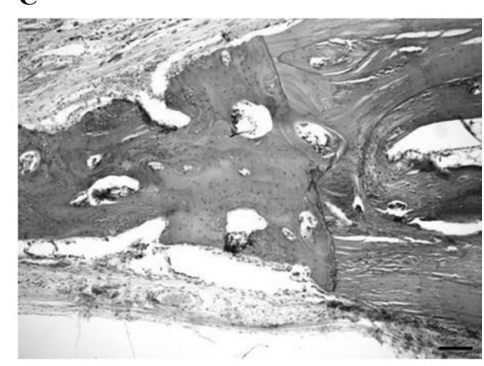

$\mathbf{F}$

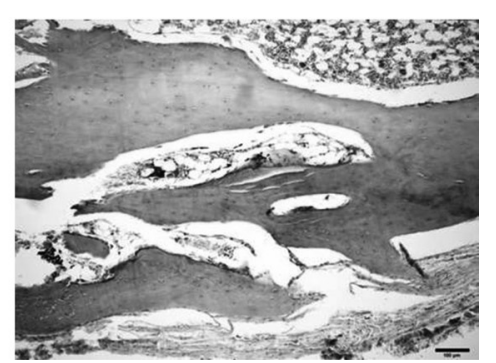

Fig. 6. Histologic section (original magnification X 100, hematoxylin-eosin stain). (A) Unfilled control 4 weeks after surgery. (B) Collagen membrane at 4 weeks after surgery. (C) 3\% 4-HR plus silk membrane at 4 weeks after surgery. (D) Unfilled control 8 weeks after surgery. (E) Collagen membrane at 8 weeks after surgery. (F) 3\% 4-HR plus silk membrane 8 weeks after surgery. 4-HR; 4-hexylresorcinol

total new bone was $31.1 \pm 7.39 \%$ in the unfilled group, $36.31 \pm$ $5.34 \%$ in the CM group, and $38.82 \pm 4.12 \%$ in the $3 \% 4-\mathrm{HR}$ plus SM group (Fig. 6). While these differences were not statistically significant, the average of total new bone in the 3\% 4-HR plus SM group was slightly higher than the CM and unfilled groups. Well-organized lamella bony islands were formed in the 3\% 4-HR plus SM group at both 4 weeks and 8 weeks (Fig. 6C and F).

\section{Discussion}

Silk is used for many medical materials. In a previous study we showed that using SFM as a barrier had a positive effect on GBR in a bone defect. SFM contributed to new bone formation in a rabbit calvarial defect model compared to the uncovered control (Song et al. 2011). However, clinical application of SFM is difficult because of its fragility and rigidity (Lee et al. 2012). In this study we applied a new form of SM that showed superior elasticity.

When the GBR technique is applied to the oral cavity the membrane can be exposed during healing which can lead to bacterial infection and GBR failure (Sanctis et al. 1996; Ling et al. 2003). Therefore, it is important to keep the surgical site free of contamination in order to prevent bacterial infection. Lee et al. used tetracycline as a membrane ingredient to prevent bacterial infection in the GBR area (Sánchez et al. 2004). When tetracycline-loaded silk fibroin membrane was used as a barrier in GBR of a bone defect, it showed more bone formation than silk fibroin alone or uncovered controls (Lee et al. 2012). Because 4-HR has been used as an antiparasitic and antiseptic agent (Rabbani et al. 1985) and was shown to be safe (Iyidogan et al. 2004), we incorporated it as an ingredient in a GBR 
membrane in order to prevent bacterial infection and promote bone regeneration in a rabbit calvarial defect model.

FT-IR analysis showed that SM had a $\beta$-sheet conformation and a random coil structure. In 3\% 4-HR plus SM the absorption bands of pure 4-HR were seen at 2,960, 2920, $2860 \mathrm{~cm}^{-1}$, and the amide bands of SM were maintained. XRD analysis of 3\% 4-HR plus SM, showed that 4-HR did not affect to the crystallinity of SM, indicating that 4-HR was stably blended within the membrane and did not change the structure of SM.

In this study, 3\% 4-HR plus SM was compared with CM. The results of $\mu$-CT analysis 4 weeks after surgery showed greater $\mathrm{BV}$ in the 3\% 4-HR plus SM group than in the unfilled control $(\mathrm{P}=0.031)$. While the mean $\mathrm{BV}$ of the $3 \% 4$-HR plus $\mathrm{SM}$ group was greater than in the CM group, the difference was not statistically significant. Histology demonstrated that well-organized lamella bone had formed at the cutting margins of the calvarial defect in the 3\% 4-HR plus SM group (Fig. 3C). Therefore, we concluded that 3\% 4-HR plus SM properly maintained the space and prevented soft tissue penetration.

The results of bone regeneration in the 3\% 4-HR plus SM group 4 weeks after surgery can be explained by the following observations. 4-HR has been used as an ingredient in antiseptic solutions and topical agents for infected skin and mucosa. Because 4-HR has disinfectant properties (Kim et al. 2012), it may contribute to preventing infection during the wound healing period. An in vitro study showed that coating the surface of an implant with a combination of hydroxyapatite and 4-HR leads to more cellular attachments and greater spreading than coating with hydroxyapatite alone (Kim et al. 2011a). 4-HR might increase the migration and attachment of osteogenic cells to the implant surface and provide assistance to initial bone formation by increasing cellular adhesion. 4-HR is known to inhibit the nuclear factor (NF)- $\kappa \mathrm{B}$ pathway (Kim et al. 2011b). Therefore, 4-HR may inhibit osteoclastogenesis. When a graft with a combination of 4-HR and hydroxyapatite was tested in a rabbit calvarial defect, it showed more initial bone volume than the unfilled control (Kim et al. 2012).

The principle of GBR is based on guided tissue regeneration (GTR) (Retzepi et al. 2010). The GTR technique employs a membrane to prevent downgrowth of highly proliferative gingival epithelial cells and promote migration of osteogenic cells and periodontal ligament cells into the osseous defect (Rothamel et al. 2005). Success of the GTR/GBR technique is largely determined in the early stages of healing. In an in vitro study of rat palatal gingiva, migration and downgrowth of the epithelium occurred after 2 weeks of GBR (Kodama et al. 1989; Minabe et al. 1989). Migration of bone and periodontal ligament cells to a bone defect reaches peak activity 2 to 7 days after initiating GBR and then decreases to almost normal levels 21 days later (Iglhaut et al. 1988). Therefore, bone formation cells might migrate to the bone defect for 3 to 4 weeks following surgery. During this initial period, the membrane should maintain a space for bone growth and prevent infiltration of soft tissue cells. Considering the importance of the initial healing period in the GBR technique, 3\% 4-HR plus SM was an effective barrier for 4 weeks despite the fact that there was no statistically significant difference in BV 8 weeks after surgery.

In this study, there were no statistically significant differences in BV among the 3 groups 8 weeks after surgery (Table I). While effectiveness might be seen in the initial healing period, the effect of 4-HR on bone formation does not persist for 8 weeks. Further study is required to understand the 8 -week results.

\section{Conclusion}

In conclusion, $\mu-\mathrm{CT}$ analysis and histologic analysis showed that the 3\% 4-HR plus SM group had more bone formation 4 weeks after surgery when compared to that of CM and unfilled control groups. 3\% 4-HR plus SM showed successful early bone regeneration in a rabbit calvarial defect model.

\section{Acknowledgements}

This study was supported by a grant from the Next-Generation BioGreen21 Program (No. PJ009013), Rural Development Administration, Republic of Korea.

This study was announced at 55th conference of the Korean Society of Sericultural Science

\section{Reference}

Altman GH, Diaz F, Jakuba C, Calabro T, Horan RL, Chen J et al. (2003) Silk-based biomaterials. Biomaterials 24, 401-416. 
Arai T, Freddi G, Innocenti R, Tsukada M (2003) Biodegradation of Bombyx mori silk fibroin fibers and films. J Appl Polm Sci 91, 23832390.

Aramwit P, Kanokpanont S, De-Eknamkul W, Srichana T (2009) Monitoring of inflammatory mediators induced by silk sericin. J Biosci Bioeng 107, 556-561.

Buser D, Dula K, Hess D, Hirt HP, Belser UC (2007) Localized ridge augmentation with autografts and barrier membranes. Periodontol 2000 19, 151-163.

Cao Y, Wang B (2009) Biodegradation of silk biomaterials. Int J Mol Sci $10,1514-1524$.

Dal Pra I, Freddi G, Minic J, Chiarini A, Armato U (2005) De novo engineering of reticular connective tissue in vivo by silk fibroin nonwoven materials. Biomaterials 26, 1987-1999.

Gosline JM, DeMont ME, Denny MW (1986) The structure and properties of spider silk. Endeavour 10, 37-43.

Gotfredsen K, Nimb L, Buser D, Hjørting-Hansen E (1993) Evaluation of guided bone generation around implants placed into fresh extraction sockets: an experimental study in dogs. J Oral Maxillofac Surg 51, 879-884.

Gottlow J (1993) Guided tissue regeneration using bioresorbable and non-resorbable devices: initial healing and long-term results. J Periodontol 64, 1157-1165.

Iglhaut J, Aukhil I, Simpson D, Johnston M, Koch G (1988) Progenitor cell kinetics during guided tissue regeneration in experimental periodontal wounds. J Periodontal Res 23, 107-117.

Imbronito AV, Todescan JH, Carvalho CV, Arana-Chavez VE (2002) Healing of alveolar bone in resorbable and non-resorbable membraneprotected defects. A histologic pilot study in dogs. Biomaterials 23, 4079-4086.

Iyidogan N, Bayındırlı A (2004) Effect of L-cysteine, kojic acid and 4-hexylresorcinol combination on inhibition of enzymatic browning in Amasya apple juice. Journal of Food Engineering 62, 299-304.

Kim J, Kim CH, Park CH, Seo JN, Kweon HY, Kang SW, Lee KG (2010) Comparison of methods for the repair of acute tympanic membrane perforations: silk patch vs. paper patch. Wound Rep Reg 18, 132-138.

Kim MK, Park YT, Kim SG, Park YW, Lee SK, Choi WS (2012) The effect of a hydroxyapatite and 4-hexylresorcinol combination graft on bone regeneration in the rabbit calvarial defect model. J Korean Assoc Maxillofac Plast Reconstr Surg 34, 377-383.

Kim S-G, Hahn B-D, Park D-S, Lee Y-C, Choi E-J, Chae W-S et al. (2011a) Aerosol deposition of hydroxyapatite and 4-hexylresorcinol coatings on titanium alloys for dental implants. J Oral Maxillofac Surg
69, e354-e363.

Kim S-G, Lee S-W, Park Y-W, Jeong J-H, Choi J-Y (2011b) 4-hexylresorcinol inhibits NF- $\mathrm{kB}$ phosphorylation and has a synergistic effect with cisplatin in KB cells. Oncol Rep 26, 1527-1532.

Kim UJ, Park J, Li C, Jin HJ, Valluzzi R, Kaplan DL (2004) Structure and properties of silk hydrogels. Biomacromolecules 5, 786-792.

Kodama T, Minabe M, Hori T, Watanabe Y (1989) The effect of various concentrations of collagen barrier on periodontal wound healing. J Periodontol 60, 205-210.

Kozubek A, Tyman JHP (1999) Resorcinolic lipids, the natural nonisoprenoid phenolic amphiphiles and their biological activity. Chem Rev 99, 1-26.

López-Caballero M, Montero P, Gómez-Guillén M (2007) Spraying of 4-hexylresorcinol based formulations to prevent enzymatic browning in Norway lobsters (Nephrops norvegicus) during chilled storage. Food chemistry 100, 147-155.

Lee SW, Park YT, Kim SG, Kweon HY, Jo YY, Lee HS (2012) The effects of tetracycline-loaded silk fibroin membrane on guided bone regeneration in a rabbit calvarial defect model. J Korean Assoc Maxillofac Plast Reconstr Surg 34, 293-298.

Ling LJ, Hung SL, Lee CF, Chen YT, Wu KM (2003) The influence of membrane exposure on the outcomes of guided tissue regeneration: clinical and microbiological aspects. J Periodontal Res 38, 57-63.

Minabe M, Kodama T, Kogou T, Tamura T, Hori T, Watanabe Y, Miyata $\mathrm{T}$ (1989) Different cross-linked types of collagen implanted in rat palatal gingiva. J Periodontol 60, 35-43.

Minoura N, Tsukada M, Nagura M (1990) Physico-chemical properties of silk fibroin membrane as a biomaterial. Biomaterials 11, 430-434.

Rabbani G, Gilman R, Kabir I, Mondel G (1985) The treatment of Fasciolopsis buski infection in children: a comparison of thiabendazole, mebendazole, levamisole, pyrantel pamoate, hexylresorcinol and tetrachloroethylene. Trans R Soc Trop Med Hyg 79, 513-515.

Rakhmatia YD, Ayukawa Y, Furuhashi A, Koyano K (2013) Current barrier membranes: titanium mesh and other membranes for guided bone regeneration in dental applications. J Prosthodont Res 57, 3-14.

Retzepi M, Donos N (2010) Guided bone regeneration: biological principle and therapeutic applications. Clin Oral Impl Res 21, 567-576.

Rothamel D, Schwarz F, Sager M, Herten M, Sculean A, Becker J (2005) Biodegradation of differently cross-linked collagen membranes: an experimental study in the rat. Clin Oral Impl Res 16, 369-378.

Sánchez AR, Rogers RS, Sheridan PJ (2004) Tetracycline and other tetracycline-derivative staining of the teeth and oral cavity. Int J Dermatol $43,709-715$. 
Sanctis MD, Zucchelli G, Clauser C (1996) Bacterial colonization of bioabsorbable barrier material and periodontal regeneration. J Periodontol 67, 1193-1200.

Schenk R, Buser D, Hardwick WR, Dahlin C (1994) Healing pattern of bone regeneration in membrane-protected defects: a histologic study in the canine mandible. Int J Oral Maxillofac Implants 9, 13.

Sofia S, McCarthy MB, Gronowicz G, Kaplan DL (2000) Functionalized silk-based biomaterials for bone formation. J Biomed Master Res 54, 139-148.

Song JY, Kim SG, Lee JW, Chae WS, Kweon HY, Jo YY et al. (2011)
Accelerated healing with the use of a silk fibroin membrane for the guided bone regeneration technique. Oral Surg Oral Med Oral Pathol Oral Radiol Endod 112, e26-e33.

Um IC, Kweon HY, Park YH, Hudson S (2001) Structural characteristics and properties of the regenerated silk fibroin prepared from formic acid. Int J Biol Macromol 29, 91-97.

Young C, Damon SR (1927) Laboratory: the use of hexylresorcinol in the treatment of typhoid carriers. Am J Public Health (N Y) 17, 279280. 